\title{
Non-Rigid Registration Techniques for Automatic Follow-up of Lung Nodules
}

\author{
Ayman El-Baz, Seniha E. Yuksel, Salwa Elshazly, Aly A. Farag* \\ Computer Vision and Image Processing Laboratory, \\ University of Louisville, Louisville, KY, 40292 \\ Contact author:farag@ cvip.uofl.edu \\ www.cvip.uofl.edu
}

\begin{abstract}
Our long term research goal is to develop an automatic approach for early detection of lung nodules that may lead to lung cancer. This paper focuses on the monitoring of the progress (growth or shrinking) of lung nodules in successive chest low dose CT (LDCT) scans of a person using non-rigid registration. The overall nodule detection approach consists of four main steps: 1) Extraction of lung region by modeling the gray level distribution of LDCT slices using a linear combination of Gaussians, 2) Classifying the lung region into homogenous tissues, arteries, veins and lung nodules, 3) Applying rigid registration using a combination of segmentation information and mutual information as a similarity measure, and 4) Compensating for the effects of heartbeats and respiration using non-rigid registration. Our earlier work addressed a number of steps in this protocol; this paper focuses on the registration step. We show that proper registration could lead to precise identification of the progress of the lung nodules.
\end{abstract}

Keywords: Motion compensation, lung nodules, segmentation, mutual information, registration.

\section{Introduction}

Follow-up of lung nodules over time is an important step in computer aided diagnosis. Approaches for the early detection of lung cancer consist of two main steps: 1) Early detection of lung nodules from either low dose spiral computed tomography (LDCT) or X-ray, and 2) Tracking the behaviour of detected lung nodules over time. In our previous work we introduced a robust system for detecting the lung nodules from LDCT scans using $2 \mathrm{D}$ and $3 \mathrm{D}$ deformable models [2]. In this paper we will concentrate on tracking the behaviour of these nodules.

In detection of lung cancer, the key point is to track the temporal behaviour of the nodules in LDCT images; since, of the suspicious nodules detected by segmentation approaches, the ones that enlarge in time have the higher possibility of being malignant.

Tracking the temporal behaviour of a nodule is a complicated task because of the change in the patient's position at each data acquisition, as well as the effects of heart beats and respiration of the patient. In order to get accurate measurements about the progress of lung nodules over the time, all this motion should be compensated by registering $\mathrm{CT}$ data sets taken at different time periods with each other. In the literature, many methods have been described for the medical image registration problem (see [3]), 
and also for the compensation of the lung motion (see [4]). Below, we will give some examples of previous work on CT lung images registration.

For the follow-up of small nodules, Brown et al. [5] developed a patient-specific model with $81 \%$ success in 27 nodules. Ko et al. [6] used the center mass point of the structures and applied rigid and affine image registration techniques with $96 \%$ success in 58 nodules of 10 patients.

To account for the non-rigid motion and deformation of the lung, Woods et al. [7] developed an objective function using an anisotropic smoothness constraint and a model based on continuum mechanics. Wood's algorithm required the detection and registration of feature points as explained in [8], and then interpolating the displacement by the model of continuum mechanics. In Wood's study, the difference of the estimated and real volumes was calculated to be $1.6 \%$. In 2003, Dougherty et al. [9] developed an optical flow method, a model-based motion estimation technique for estimating first a global parametric transformation and then local deformations. This method allowed the alignment of serial CT images with a 95\% correlation. Another optical flow analysis approach was developed by Naqa et al. 2003 [10], where the optical flow analysis was combined with the information of a spirometer, a device measuring the airflow into and out of the lungs to track the breathing motion automatically. The spirometry approach used in Naqa's study was based on the reconstruction techniques for 4D CT during free breathing proposed by [11].

There are also studies using CT lung images for pulmonary registration. For this purpose, Zhang et al. [12] used a standard lung atlas to analyze the pulmonary structures in $\mathrm{CT}$ images. This atlas is registered to new images by a $3 \mathrm{D}$ surface based registration technique composed of global transformation and local elastic transformations. Li et al. [13] used feature points for correspondence and landmark \& intensity based registration algorithms to warp a template image to the rest of the lung volumes.

\section{Proposed Approach}

In this paper we introduce a new approach to track the behaviour of lung nodules, over time, for a certain subject. The proposed approach consists of four main steps. These steps are:

1) Extraction of the lung region from other anatomical structures that surround the lung tissue such as chest bones.

2) Classifying the lung tissues to homogeneous tissues (appear in black in LDCT) and arteries, veins and lung nodules.

3) Performing rigid registration to capture the global motion of the patient using affine transformation.

4) Performing B-splines based non-rigid registration to compensate the lung deformation due to heart beats and respiration of the patient.

For the $3^{\text {rd }}$ and $4^{\text {th }}$ steps, we used normalized mutual information (MI) based on based on both the gray level intensity and the tissue label (homogeneous tissues, arteries, veins, and lung nodules) as a similarity measure to get the best transformation. By modifying the mutual information calculations using the tissue labels, we were able to benefit from segmentation results in the registration process. 


\subsection{Extraction of Lung Region and Classifying the Lung Tissues}

As we mentioned above, the first step of the proposed approach is to extract the lung region from the surrounding anatomical structure. To accomplish this step accurately, we model the gray level distribution in each data set using a linear combination of Gaussians (LCG) distribution having positive and negative components. The parameters of the LCG such as mean variance are estimated using our novel EM algorithm as described in [14]. By using both positive and negative Gaussians components we can get very accurate approximation for the gray level distribution for each data set as shown in Figure 1. Figure 2(a) shows a typical chest slice from a spiral LDCT scan. Figure 2(b) shows the results of lung region extraction using the LCG approach. After we extract the lung region we can use the LCG model to model the gray level distribution within the lung region as shown in Figure 1(b). The results of classified lung tissues using the LCG model is shown in Fig. 2(c), the homogeneous tissues (shown in red) and arteries, veins, and lung nodules (shown in green).
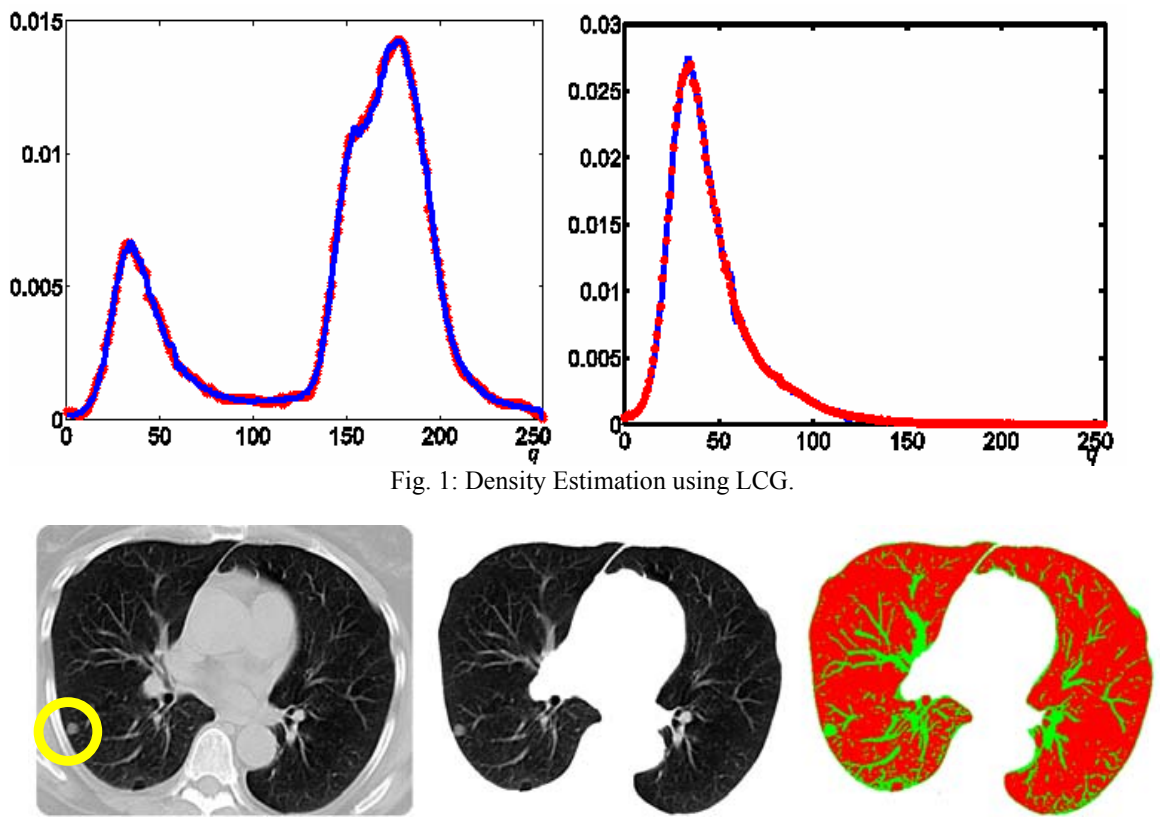

Fig. 2: The first two steps of the proposed system to classify the tissue

\subsection{Rigid Registration}

The goal of the rigid registration step is to capture the global motion of the patient. A simple choice is an affine transformation which handles the rotations, translations, scaling and shearing of the lung. However, we have integrated the segmentation results 
into our rigid registration scheme by a simple addition of the label information, which increased the accuracy of the registration. The proposed rigid algorithm is as follows:

1. Select sample data set from the first volume, $A$. Represent each sample by two values, the gray level and the label.

2. Initialize a transformation function $T$.

3. Multiply the sample from volume $A$ by the transformation function $T$.

4. Use the k-nearest sampling method to get the corresponding points from volume $B$.

5. Use the genetic algorithm to find the transformation function $T$ that maximizes the normalized mutual information based on gray level and tissue label information with the pseudo code as given below.

In the pseudocode below, we have two images named Fixed_Image and the Moving_Image, and their segmented (labeled) images Fixed_Image_Label and Moving_Image_Label. The transformation is applied on the Moving_Image and the pixel is introduced into the joint histogram calculations only if the transformed pixel has the same label as the fixed image.

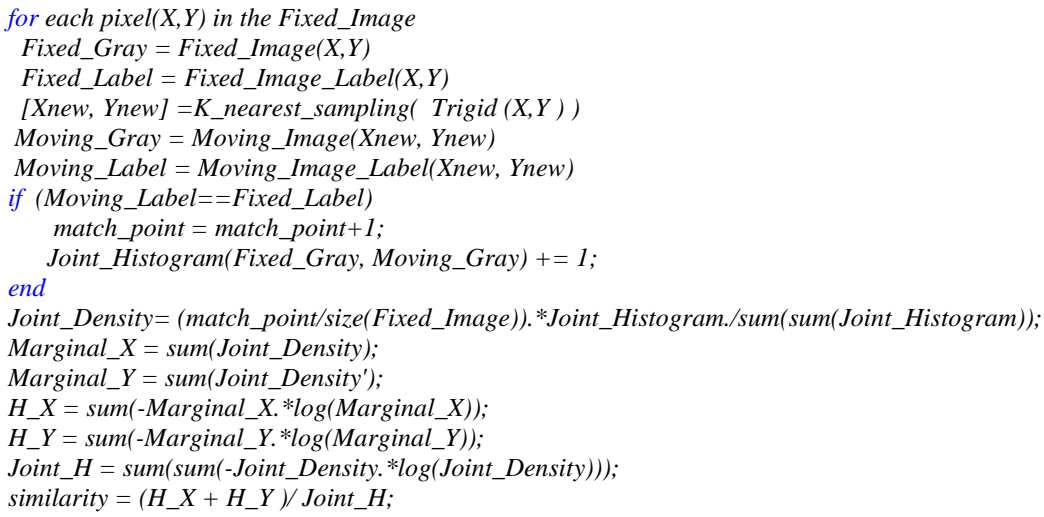

\subsection{Non-Rigid Registration}

The rigid transformation captures only the global motion of the lung. An additional transformation is required, which models the local deformation of the lung due to the motion that comes from the heart beats and breathing. The nature of the local deformation of the lung can vary significantly across patients and with age. Therefore, it is difficult to describe the local deformation via parameterized transformations. Instead, we chose a free form deformation (FFD) model based on B-splines [15], which is a powerful tool for modelling 3-D deformable objects. The basic idea of FFD's is to deform an object by manipulating an underlying mesh of control points. The resulting deformation controls the shape of the 3-D object and produces a smooth and continuous transformation. Fig. 3 shows the result of these rigid and non-rigid transformations. 


\subsection{Nodule Evaluation}

After the two volumes of different time instants are registered, the task is to find out if the nodules are growing or not. For this purpose, after registration, we are reslicing the volume by cubic interpolations and segmenting the nodules once again, using the method of El-Baz et al. [2]. Once the nodules are segmented in the original and registered image sequences, the volumes of the nodules are calculated using the $\Delta x, \Delta y$ and $\Delta z$ values from the scanner. In our studies, these values are $0.4 \mathrm{~mm}, 0.4 \mathrm{~mm}, 2 \mathrm{~mm}$ respectively. At the last step, drawing a progress curve would show the size of the nodule in time. Although a nodule growing in time doesn't mean that it is cancer, the slope of this curve helps to understand the acuteness of the disease. After the registration, we are taking two slices from each volume by cubic interpolation as a sampling process. Figure 4 shows these slices using a checkerboard effect for visualization. The smooth continuity of the boundary and the arteries show the quality of the proposed approach. Table 1 shows the detected changes in the lung nodules over nine months.

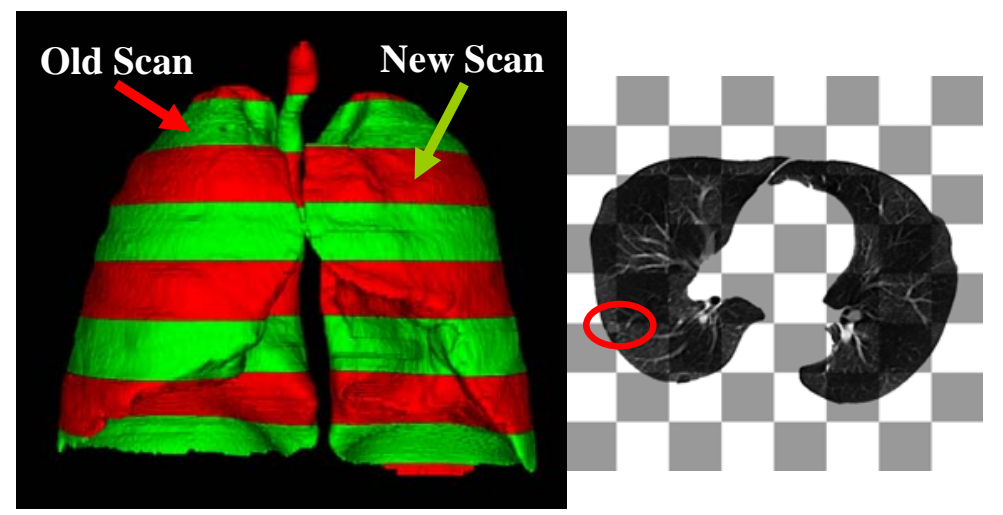

Fig. 3: Registration of the old and new scans of the lung Fig. 4: 2D slice to show the quality of the approach

\section{Conclusion}

For the detection of growing lung nodules, accurate registration is crucial. In this paper, we introduced a new approach for the registration of spiral CT images for cancer detection, and using this algorithm, a progress curve of a nodule would give very important information about the severity of the disease.

However, B-spline based non-rigid registration algorithms cannot handle large deformations, and therefore, instead of using a rectangular grid with B-splines, our future work will include non-rigid registration based on level sets.

\section{References}

[1] P. M. Boiselle and C. S. White (Eds.) New Techniques in Thoracic Imaging. M. Dekker, New York, 2002.

[2] A.A. Farag, A. El-Baz, G.Gimelfarb, R Falk, and S.G. Hushek, "Automatic detection and recognition of lung abnormalities in helical CT images using deformable templates," Lecture Notes in Computer Science, 
Table 1: The results of the proposed follow up registration algorithm for four patients over nine months

\begin{tabular}{|c|c|c|c|c|c|}
\hline Parameters & Subject \#1 & Subject \#2 & Subject \#2 & Subject \#3 & Subject \#4 \\
\hline Old Scan & & & & & \\
\hline $\begin{array}{l}\text { Scan after } 9 \\
\text { months }\end{array}$ & & & & & \\
\hline $\begin{array}{l}\text { Changes are } \\
\text { shown in red }\end{array}$ & & & & & \\
\hline $\begin{array}{l}\text { Volume } \\
\text { change in } \\
\mathrm{mm}^{3}\end{array}$ & $0.84 \mathrm{~mm}^{3}$ & $0.3872 \mathrm{~mm}^{3}$ & $0.7877 \mathrm{~mm}^{3}$ & $0.5407 \mathrm{~mm}^{3}$ & $0.6542 \mathrm{~mm}^{3}$ \\
\hline
\end{tabular}

vol. 3217, pp. 131-139, September, 2004. (Proc. of International Conference on Medical Image Computing and Computer-Assisted Intervention, MICCAI-2004, September 26-29 2004, pp. 856-864).

[3] J. B. A. Maintz and M. A. Viergever, "A Survey of Medical Image Registration," Medical Image Analysis, vol.2, pp.1-36, 1998.

[4] J.P. Ko and D.P. Naidich, "Computer-aided diagnosis and the evaluation of lung disease", Journal of thoracic imaging, pp. 19:136, 2004.

[5] M.S. Brown, M.F. McNitt-Gray, N.J. Mankovich et al., "Method for segmenting chest CT image data using an anatomical model: preliminary results.” IEEE Trans Med Imaging, vol. 20, pp. 242-1250, 2001.

[6] J. P. Ko, and M. Betke, "Chest CT: automated nodule detection and assessment of change over timepreliminary experience” Radiology, 218: 267-273, 2001.

[7] K. Woods, L. Fan, C. W. Chen, and Y. Wang, "Model Supported Image Registration and Warping for Change Detection in Computer-aided Diagnosis," Applied Imagery Pattern Recognition (AIPR) Annual Workshops 2000, Washington DC, 2000.

[8] L. Fan and C. W. Chen, "An integrated approach to 3D warping and registration from lung images," Proceedings of SPIE Conf. Developments in X-Ray Tomography II, Denver, CO, July 1999

[9] L. Dougherty, J.C. Asmuth and W. B. Gefter, "Alignment of CT lung volumes with an optical flow method", Acad. Radiol., 10:249-254, 2003.

[10] I. E. Naqa, D.A. Low, J.O. Deasy et al., "Automated breathing motion tracking for 4D computed tomography” Nuclear Science Symposium Conference Record, 2003 IEEE, vol. 5, 19-25 Oct. 2003, pp. $3219-3222$, Vol.5

[11] D. Low et al., "A method for the reconstruction of four-dimensional synchronized CT scans acquired during free breathing" Medical Physics, Vol. 30, No. 6, June 2003.

[12] L. Zhang and J. Reinhardt, "3D pulmonary CT image registration with a standard lung atlas," in Proc. SPIE Conf. Medical Imaging, 4322, pp. 67-77, 2000.

[13] B. Li, G. E. Christensen, J. Dill, E. A. Hoffman, and J. M. Reinhardt, "3-D inter-subject warping and registration of pulmonary CT images for a human lung model” In Proc. SPIE Conf. Medical Imaging, volume 4683, pp. 324-335, San Diego, CA, 23-28 Feb. 2002.

[14] A.A. Farag, A. El-Baz, and G. Gimel'farb "Density Estimation Using Modified Expectation Maximization for a linear combination of Gaussians," Proc. of IEEE International Conference on Image Processing (ICIP- 2004), Singapore, October 24-27 2004, pp. 194-197.

[15] D. Rueckert. Non-rigid registration: Techniques and applications. In J. V. Hajnal, D. L. G. Hill, and D. J. Hawkes, editors, Medical Image Registration. CRC Press, 2001. 